\title{
An Shoulder Peak Resistant Pin Security Scheme using Concentrate Haptic Feedback
}

\author{
Kumararaja V, Sathyapriya R, Sharmila T, Sobika S,Tamilselvi V
}

\begin{abstract}
Fundamental PIN-area plans are vulnerable against recognition strikes. To overhaul the assurance from observation attacks, some discernment ambushes safe PIN-segment plans for PDAs reliant on sounds just as haptics have been proposed. In any case, none of existing observation attacks safe PIN-entry plans can achieve both incredible security and high accommodation. Here in, we propose another discernment attacks safe PIN-segment scheme, Loc-HapPIN, for contact screen contraptions giving limited haptic analysis. By using the advancement of limited haptic info, the convenience and the insurance from discernment ambushes are improved. In addition, the customer can pick the viability security setting sensible for them.
\end{abstract}

\section{INTRODUCTION}

Printed passwords have been the most for the most part used approval procedure for a significant long time. Included numbers and upper-and lower-case letters, abstract passwords are seen as adequately ready to contradict against creature control attacks. In any case, a strong scholarly mystery express is hard to recollect and review. In like manner, customers will when all is said in done pick passwords that are either short or from the word reference, rather than unpredictable alphanumeric strings. Unmistakably increasingly horrendous, it's definitely not an extraordinary case that customers may use only a solitary username and mystery express for different records. As shown by an article in Computer world, a security bunch at an extensive association ran a framework mystery key saltine and incredibly split generally $80 \%$ of the delegates' passwords inside 30 seconds. Abstract passwords are sometimes questionable due to the inconvenience of keeping up strong ones. Distinctive graphical mystery key affirmation plans were made to address the issues and deficiencies related with printed passwords. In light of a couple of examinations, for instance, those in individuals have a better limit than recall pictures with whole deal memory (LTM) than verbal depictions. Picture based passwords were ended up being less requesting to recall in a couple of customer ponders. In this way, customers can set up a marvelous check mystery state and are fit for reviewing it after a long time paying little respect to whether the

Revised Manuscript Received on April 12, 2019.

Kumararaja V,Department of computer science and engineering V.S.B. Engineering College, Karur, Tamilnadu, Pin-639111 (Email: kumarcs@gmail.com)

Sathyapriya R,Department of computer science and engineering V.S.B. Engineering College, Karur, Tamilnadu, Pin-639111

Sharmila T,Department of computer science and engineering V.S.B. Engineering College, Karur, Tamilnadu, Pin-639111

Sobika S,Department of computer science and engineering V.S.B Engineering College, Karur, Tamilnadu, Pin-639111

Tamilselvi V,Department of computer science and engineering V.S.B Engineering College, Karur, Tamilnadu, Pin-639111
Keywords:

memory isn't started once in a while. In any case, most of these image based passwords are exposed against shoulder surfing attacks (SSAs). This kind of attack either uses direct discernment, for instance, paying special mind to someone's shoulder or applies video getting frameworks to get passwords, PINs, or other delicate individual information. Standard mystery key structures are regularly message based and frail against various strikes, including shoulder surfing or word reference ambushes. As more customers and affiliations get settled with these attacks, the need to address the security of such systems has in like manner grown rapidly. Mystery state approval systems should bolster less obvious and strong passwords while keeping up update capacity and security. Alphanumerical usernames and passwords are most normally used for customer affirmation. One of the impediments of picking such chart is passwords could be basically conjectured. It is uncommon to not consider that in case a mystery expression is hard to figure, by then generally hard to recall. Alphanumeric passwords are moreover disposed to dictionary strikes. Due to the issue with recalling unpredictable arrangement of characters, most customers as often as possible settle on an ordinary word or a name without understanding that their picked passwords can be monster compelled generally in a very less time.

Individuals can review pictures much better than the substance. This reality has been abused to propose graphical mystery express plots as a functional alternative as opposed to content based plans. In graphical mystery key plans, pictures are used instead of alphanumeric characters. Customer must review a ton of pictures to precisely login. The unquestionable hindrance of such an arrangement is having a huge dictionary of such novel pictures set away in a memory anyway if the amount of possible pictures is enough tremendous, the possible mystery key space of a graphical mystery word clearly offer better security from vocabulary ambushes. Another shortcoming is that these graphical plans are slanted to hold up under surfing attacks.

In shoulder surfing, an enemy endeavors tofigure the mystery word by particularly looking customer login their screens. A mystery word easy to review for an authentic customer is in like manner imperative for a foe.

In nearness without the most ideal usage of security or verifies Mobile Phones which prompts vulnerability of taking others singular information. This individual information incorporates mishandling others photo's, dealing with a record nuances, getting some fundamental reports being manhandled by others without real security plan. The issue of security is winding up dreadful on account of cutting edge cell phone usage.

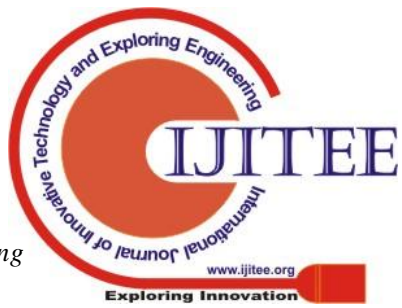


This endeavor is a compact application based undertaking to improve security. "stick security conspire utilizing haptic input" is a simple to utilize programming application. The inspiration driving this endeavor is to give a prevalent security, an item game plan that passes on an adaptable, secure, and trustworthy application that keeps up and manages the application nuances. The going with Document will graph the component of the "stick security plot utilizing haptic criticism" and the essentials that the endeavor will hold quick to working up the item for the customer security reason.

\section{RELATED WORKS}

Various graphical mystery key plans are proposed with an objective to save memory, work brisk and less slanted to manage surfing strikes. One of the early graphical check plans is proposed by Dhamija and Perrig[4]. Their technique relies upon hash portrayal where the server needs to store the seeds of the portfolio photos of all customers in plaintext. Furthermore, discretionary picture decision from a gigantic database is a dull strategy and plans have been proposed to capably store and pick pictures. In a system called "Passface", the customer is requested to pick four pictures from human appearances. The affirmation dispense with shows 9 faces of which eight are impersonation and one is the as of late selected from four. The customer sees and snaps wherever on the known face. This strategy is comprehensively huge over long intervals [1]. In any case, the genuine drawback of such an arrangement is a trademark propensity to pick faces subject to sexual introduction, race or social inclinations. This makes the mystery key somewhat obvious. Brostoff and Sasse [9] similarly finished an examination which revealed that Passface count was less utilized by customers in light of the way that the framework took a widely inclusive system to affirm than substance based passwords. This methodology is broadly critical over long intervals. In any case, the huge disservice of such an arrangement is acharacteristic inclination to pick faces subject to sexual introduction, race or social propensities. This makes the mystery expression somewhat obvious. Brostoff and Sasse [9] in like manner finished an examination which revealed that Passface count was less utilized by customers in light of the way that the system took a widely inclusive methodology to approve than substance based passwords.

\section{Existing System}

A one of a kind imprint scanner is a kind of advancement that recognizes and affirms the fingerprints of an individual in order to give or deny access to a PC system or a physical office. In extraordinary imprint looking at plan, the lock can be successfully grabbed by other individual if we have gently kept the finger in any of the articles.

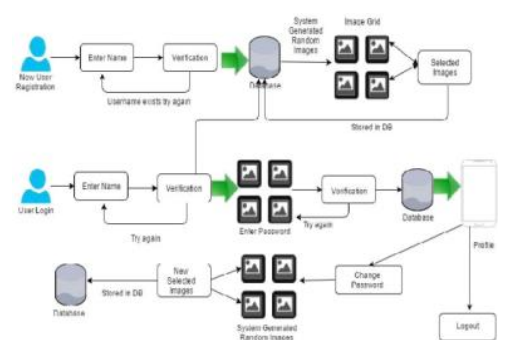

Fig: Text based Graphical Password System Diagram

A mystery word is a progression of characters used to check the identity of a customer in the midst of the confirmation technique. Passwords can contrast long and can contain letters, numbers and uncommon characters. In mystery state lock, passwords can be viably gotten using shoulder surfing strike where the other individual can without a doubt misuse the watched mystery express.

\section{PROPOSED SYSTEM\& RESULTS}

The rule thought behind this application is to improve the security. At first the customers enrolls the required fields and get the OTP. Haptic analysis structure is open to balance shoulder surfing ambushes that send OTP to any selected adaptable GSM device. The Random vibration for the each stick is made. On the off chance that somebodyendeavor to mishandle the stick their image is been catch and sent to the owner's enrolled convenient number.

The procedures that I have used for this application are according to the accompanying:

1. A pseudo-sporadic procedure is a program with number generator made for, and used in, probability and estimations applications when enormous measures of subjective digits are required. The larger pieces of these ventures produce boundless strings of single-digit numbers, usually in base 10, known as the decimal structure. Right when extensive instances of pseudo-self-assertive numbers are taken, all of the 10 digits in the set $\{0,1,2,3,4,5,6,7,8,9\}$ occurs with proportionate repeat, regardless of the way that they are not consistently scattered in the progression.

2. Pseudo Random system which can be reproducible if the estimation is found. The very nearness of the estimation, paying little respect to how perplexing, suggests that the accompanying digit can be foreseen. This has offered climb to the term pseudo-sporadic for such machine-made arrangement of digits. They are equivalent to sporadic number groupings for most applications, anyway they are not by any means selfassertive as demonstrated by the exhaustive definition.

The Haptic info is a security plot in which we need to select the required nuances and a phone number is enrolled for recovery reason and an OTP is made. An affirmation is done by sending an OTP to the selected number and a security question and answer is been set to recover the neglect mystery key. In case someone endeavor to get to it will get the general population picture and will send to the 
enrolled number. Haptic Feedback, oftentimes alluded to as essentially "haptics", is the use of the sentiment of touch in a UI arrangement to offer information to an end customer. When evading to PDAs and practically identical contraption, this all around strategies the use of vibration from the device's vibration.

The objective of my endeavor is to check a fitting application lock for different applications. This is done by sporadic vibration procedure using an unadulterated subjective variable relies upon air parameter so it can't be rehashed. This vibration is used to count the formally enrolled stick. After vibration in case you press enter the applications that are checked will be showed up.

The endeavor is about the PIN security plot using Haptic Feedback. The objective of this application is to find whether the present application locks are checked are assuredly not. This is done by self-assertive vibration using Sqlite. This is used to count the enrolled PIN with the vibration. These are the modules of PIN security scheme using Haptic analysis.

\section{Enrollment Module-1}

The customer need to enroll the normal nuances to understand that the required individual use the compact Username, mail-id and the recovery phone number is set first and set away in the Database after the summit of first page, The second page with further nuances get appeared by clicking straightaway.

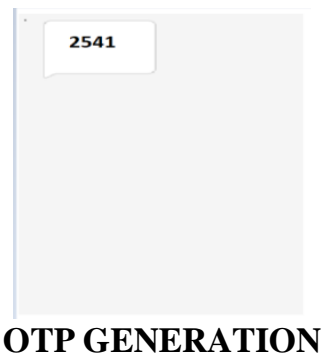

\section{Enrollment Module-2}

An OTP is sent to the enrolled number in the wake of displaying the fundamental page. Enter the OTP, if you enter any number heedlessly you will get a goof message that your OTP isn't right. You can enter the mystery key the limit of the mystery word is bound just to 4digit, the security question can be entered by the customer and the security answer is also set by the customer all of the nuances are secured in the Database by clicking straightaway.

\section{Random Vibration Technique}

From the enrolment module- 2 we have saved a mystery key for our advantage and after that there is a vibration setup. By using assorted vibration, we have to enter the PIN which depends upon the customer. Once, the stick is entered, press straightaway. In light of the vibration, the stick that we have starting at now setup matches with the continuous entered PIN is right then we go to the accompanying module that then the application is to get checked and it will appear.

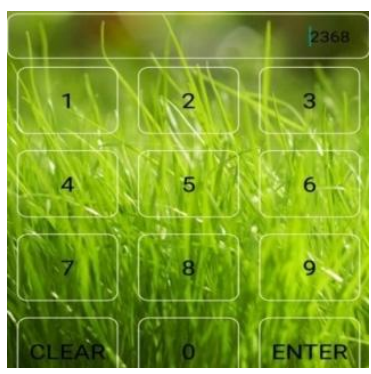

\section{Overlooked secret phrase}

If the mystery expression isn't right we will get the neglected mystery state the security question which we have enrolled is appeared disregarded mystery word enter the correct reaction for that question the mystery expression that we have saved is sent to the selected number.

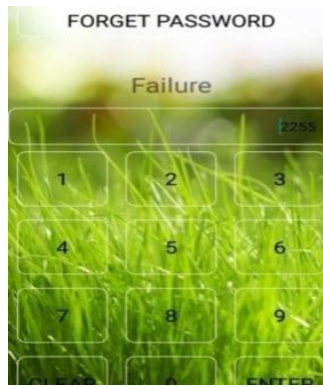

\section{Picture Capture Message}

In this module, if anyone sees our delivered one time mystery word and, by then they endeavor to retype and open any application, by then their image will be gotten and sent to enrolled convenient number. So manhandling of our flexible by others can be adequately recognized.

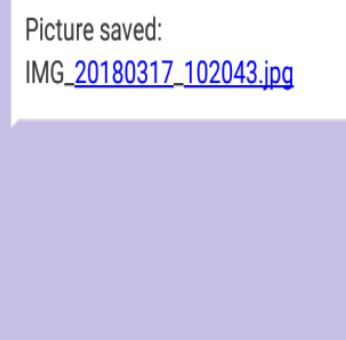

\section{CONCLUSION}

The endeavor PIN security scheme using Haptic analysis is astoundingly essential in structure and to realize. The convenient requires outstandingly low resources and works in for all intents and purposes all structures and its interface is straightforward. It fuses selection of the customer, by then the discretionary vibrations are counted which is incorporated with adequately existing PIN and new mystery key is created as a general rule. The delivered new mystery key is been formed to open an application. Same Mobile application can be made for others Mobile working structures, for instance, Windows, iOS, etc. Existing writing computer programs is created for cell phones of Android 
working structure with treat interpretation and underneath. Same application can be made for higher android variation. In our present endeavor, we have included security only for the calculator application. In future, the customer could prepared to pick any applications available in his wireless, so the picked applications can be checked.

\section{REFERENCES}

1. S. Sood, A. Sarje, and K. Singh, "Cryptanalysis of secret key validation plans: Current status and key issues," in Strategies and Models in Computer Science, 2009. ICM2CS 2009. Continuing of International Conference on, Dec 2009, pp. $1-7$.

2. A. Paivio, T. Rogers, and P. Smythe, "For what reason are pictures simpler to review than words?" Psychonomic Science, 1968. what's more, Models in Computer Science, 2009. ICM2CS 2009. Continuing of International Conference on, Dec 2009, pp. 1- 7.

3. A. De Angeli, M. Coutts, L. Coventry, G. Johnson, D. Cameron, and M. Fischer, "Vip: a visual way to deal with client verification," in Proceedings of the Working Conference on Advanced Visual Interfaces. ACM, 2002, pp. 316-323.

4. B. Ives, K. Walsh, and H. Schneider, "The domino impact of secret word reuse," Communications of the ACM, vol. 47, no. 4, pp. 75-78, 2004.

5. Suo, Xiaoyuan, Ying Zhu, and G. Scott Owen. "Graphical passwords: A review." Computer security applications meeting, 21st yearly. IEEE, 2005 . 